\title{
I-Suit Advanced Spacesuit Design Improvements and Performance Testing
}

\author{
David Graziosi and Ryan Lee \\ ILC Dover, Inc.
}

Copyright (C) 2003 SAE International

\begin{abstract}
The I-Suit has been tested in varying environments at Johnson Space Center (JSC). This includes laboratory mobility testing, KC-135 partial gravity flights, and remote field testing in the Mojave Desert. The experience gained from this testing has provided insight for design improvements. These improvements have been an evolutionary process since 1998 to the present. The design improvements affect existing suit components and introduce new components for systems processing and human/robotic interface. Examples of these design improvements include improved mobility joints, a new helmet with integrated communications and displays capability, and integration of textile switches for control of suit functions and tele-robotic operations.
\end{abstract}

This paper addresses an overview of I-Suit design improvements and results of manned and unmanned performance tests. The performance tests include mobility joint testing, helmet visibility testing, and system level manned testing of the improved suit components.

\section{INTRODUCTION}

The success of astronauts performing Extra-Vehicular Activity (EVA) is highly dependent on the performance of the space suit system they are wearing. Future EVA spacesuits will have to be capable of handling multiple roles. Future missions will require a spacesuit to be capable of performing zero gravity operations as well as varying partial gravity missions. The multi-role mission will require suit qualities not available in any currently operational EVA spacesuit. Future spacesuits will have to provide excellent full body mobility, be very lightweight, robust and comfortable. The I-Suit was developed to evaluate the current state of the art in EVA spacesuit design and to provide a test bed suit for evaluating future multi-role EVA. A prototype EVA spacesuit assembly was designed and fabricated under a joint NASA/ILC Dover Inc. funded contract over a 15-month period from 1997 to 1998. NASA provided input on design goals as well as several constraints on suit configuration. This spacesuit is named the I-Suit.

\section{I-SUIT DEVELOPMENT HISTORY}

ORIGINAL NASA CONTRACT

The I-Suit development was started in the summer of 1997 with a contract awarded to ILC Dover. This contract was for the development of an advanced all soft multi-bearing EVA spacesuit. ILC Dover also used its own research dollars to develop new mobility joint designs. The combined effort lead to delivery of a new advanced spacesuit prototype in approximately 15 months. Once delivered to NASA the I-Suit was tested for individual mobility joint performance. The I-Suit was also tested in a partial gravity environment in NASA's $\mathrm{KC}-135$ aircraft and in a 1-G Martian analog during NASA's Astronaut Rover Interaction field test in the Mojave Desert.

\section{REQUIREMENTS}

NASA imposed numerous configuration requirements on the first generation of I-Suit. The configuration requirements included the use of the existing Space Shuttle EMU helmet assembly and wrist bearing/disconnect, 2-bearing hip system, all soft fabric suit elements, hard waist entry design, and walking boots. NASA also imposed performance requirements on the new soft suit relating to pressure, structural loads, mobility joint torque and range of motion, and sizing capability.

\section{DESIGN CONFIGURATION}

The I-Suit final design focused equally on upper torso and lower torso mobility. The upper torso is an all-soft fabric design with waist entry. The upper torso design included an all soft single axis balanced convoluted shoulder mobility joint with scye bearing and arm bearing. The lower arm is a single axis uni-directional gored joint with wrist quick disconnect and bearing. The lower arm also has a built in sizing element and cam actuated brackets for vernier sizing. The waist mobility joint is an all-soft single axis balanced design with 
integral torso sizing. The waist joint provides for 3 inches of torso sizing. The brief is an all-soft fabric design similar in construction to the soft upper torso. The hip/thigh is a 2 bearing design with an integral single axis convoluted mobility joint. The leg design is similar to the lower arm with two integral sizing elements above and below the knee and one removable sizing element below the knee. The removable element provides large changes in sizing while the integral elements provide vernier sizing. The boot design is an integral part of the leg. The ankle joint is a convoluted design similar to the shoulder mobility joint. The boot includes a flexible sole and internal inflatable foot restraint system. An illustration of the original I-Suit configuration is shown in Figure 1.

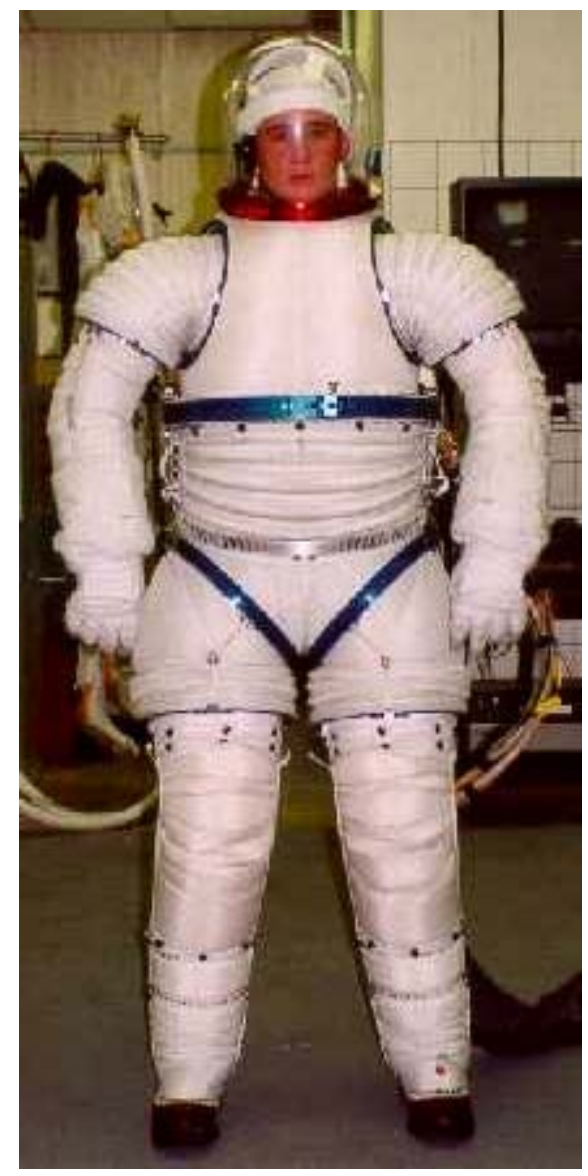

FIGURE 1

\section{GENERATION II I-SUIT}

After the delivery of the first generation of I-Suit to NASA and the completion of performance evaluations, work was begun on the design improvements. These design improvements spanned several years of development and include a new helmet system, lower torso redesign including the hip/thigh joint, boots and waist hardware. Some smaller components for the new I-Suit were also developed for evaluating small astronaut performance while retaining generic large sized hardware (bearings, helmet and disconnects). The development work also expanded to include evaluations of electronic textile applications, and information display through the integration of micro display technology and computer technology.

\section{ADVANCED HELMET DESIGN}

The new helmet was developed to improve on the limitations of the Shuttle EMU helmet used on the original I-Suit. The Shuttle helmet design has minimal internal volume that limits astronaut upward and downward visibility and can cause crewmember discomfort from repeated contact with the helmet neck ring. It is also too small to provide room for an advanced 'heads free' communication system that would include integral speakers and boom microphones. The new ISuit helmet design includes a composite wedge element, which provides a method for future life support backpack attachment and housing for life support air, water, and electrical interfaces.

The new helmet development started with the fabrication of CAD models of proposed concepts and evaluations of these models using anthropometric software. Helmet shapes included numerous circular and elliptical geometries. These evaluations included evaluations of helmet size required to properly fit a $5^{\text {th }}$ percentile female to $95^{\text {th }}$ percentile male head size. See Figure 2 .

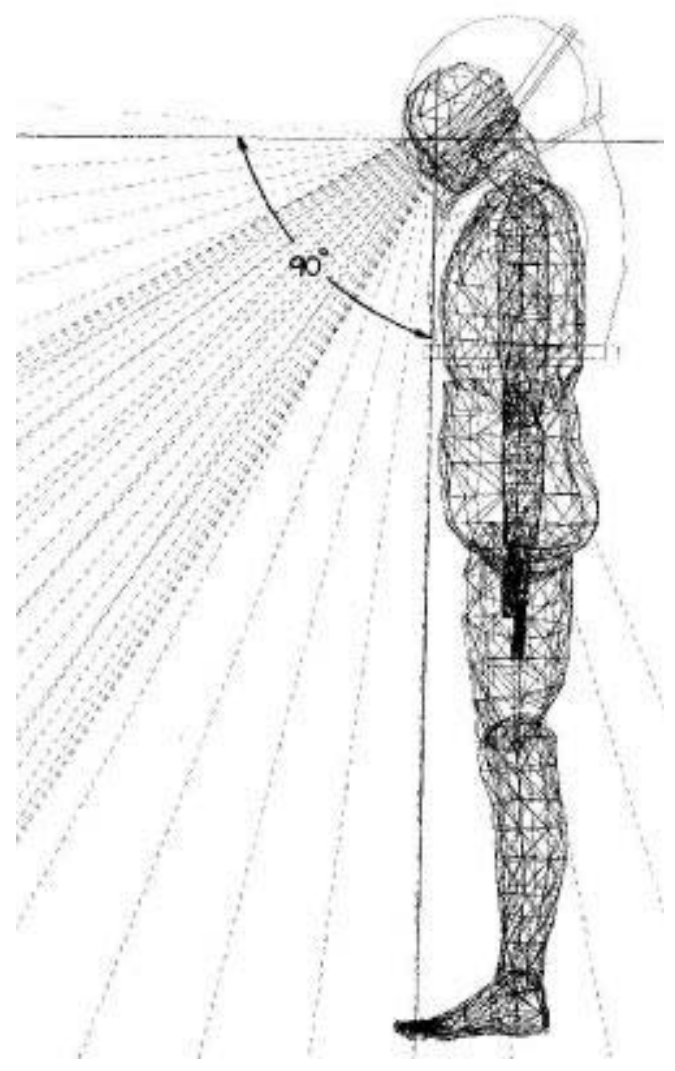

\section{FIGURE 2}

The new helmet had to provide improved upward and downward visibility over the Shuttle helmet. Once numerous concepts were down selected using CAD modeling, helmet mockups were fabricated and manned 
testing was performed to validate the theoretical data. A picture of the helmet test fixture can be seen in Figure 3.

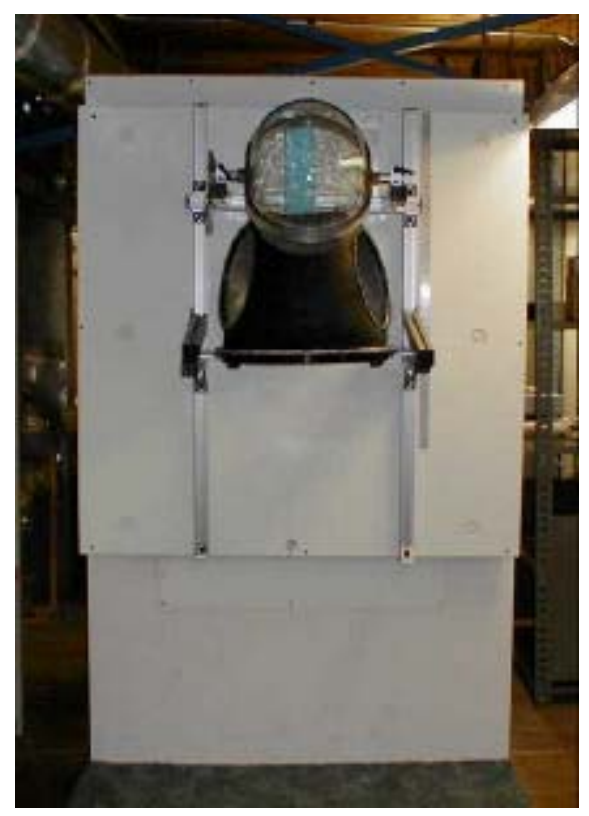

FIGURE 3

The empirical helmet testing was performed on 7 subjects. Critical subject head dimensions varied from $4^{\text {th }}$ percentile to $99^{\text {th }}$ percentile. The data shows an average improvement in upward (Superior) visibility of 25 degrees for the final version of the I-Suit helmet over the existing Shuttle helmet. Downward (Inferior) visibility showed an average improvement of 6 degrees without the chest mounted Shuttle display and control module (DCM) and 15 degrees improvement with it. Table 1 shows the results of the visibility testing.

Once the basic geometry was defined, a complete 3D model was fabricated using Pro Engineering software. This model was the starting point for integration of critical elements such as the air in plenum, head back pad, speaker housings, and microphone mounting blocks. Once these elements were designed and integrated into the model, a helmet full-scale prototype was fabricated using stereo lithography. This prototype was used to evaluate helmet vent flow and carbon dioxide washout performance. Figure 4 shows a picture of the prototype helmet design and vent flow fixture.

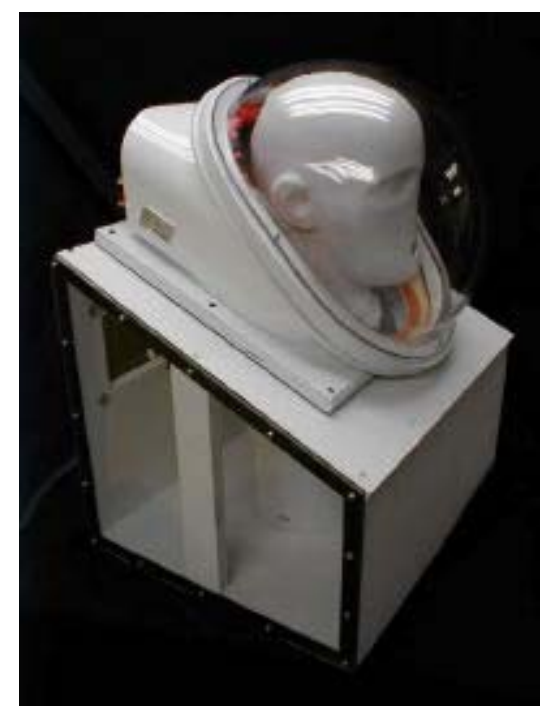

FIGURE 4

Testing performed using the prototype helmet and vent flow fixture provided critical data to modify the air-in plenum design as well as other helmet features. Once testing was completed the Pro Engineer model was updated to the final design and a fully functional prototype helmet was fabricated. Manned pressurized ISuit testing was performed at ILC Dover and Johnson Space Center to evaluate the performance of the new helmet. The helmet design passed spaceflight helmet carbon dioxide washout tests performed at NASA JSC. Test engineers and astronauts at NASA JSC found the new advanced I-Suit helmet to be a significant improvement over the existing Shuttle helmet.

HELMET VISIBILITY RANGE COMPARISON

\begin{tabular}{|l|c|c|c|c|c|c|c|c|}
\hline & $\begin{array}{c}\mathrm{H} 34 \\
\text { Pronasale- } \\
\text { Back of Head }\end{array}$ & $\begin{array}{c}\mathrm{H} 30 \\
\text { Menton- } \\
\text { Top of Head }\end{array}$ & $\begin{array}{c}\mathrm{H} 6 \\
\text { Bizygomatic } \\
\text { Breadth }\end{array}$ & $\begin{array}{c}\text { (degrees) } \\
\text { I-Suit } \\
\text { Superior }\end{array}$ & $\begin{array}{c}\text { (degrees) } \\
\text { Shuttle } \\
\text { Superior }\end{array}$ & $\begin{array}{c}\text { (degrees) } \\
\text { I-Suit } \\
\text { Inferior (No DCM) }\end{array}$ & $\begin{array}{c}\text { (degrees) } \\
\text { Shuttle } \\
\text { Inferior (No DCM) }\end{array}$ & $\begin{array}{c}\text { (degrees) } \\
\text { Shuttle } \\
\text { Inferior (DCM) }\end{array}$ \\
\hline Subject 1 & $9.2(97 \%)$ & $8.7(12 \%)$ & $20 \%$ & 118 & 96 & 84 & 76 & 67 \\
\hline Subject 2 & $9.0(90 \%)$ & $8.5(4 \%)$ & $5.0(<1 \%)$ & 135 & 115 & 87 & 85 & 75 \\
\hline Subject 3 & $9.4(99 \%)$ & $9.06(43 \%)$ & $6.0(92 \%)$ & 123 & 109 & 87 & 83 & 74 \\
\hline Subject 4 & - & --- & - & 126 & 96 & 88 & 80 & 72 \\
\hline Subject 5 & $9.2(95 \%)$ & $8.9(25 \%)$ & $5.4(15 \%)$ & 134 & 115 & 87 & 83 & 73 \\
\hline Subject 6 & $8.8(70 \%)$ & $9.1(47 \%)$ & $5.45(22 \%)$ & 140 & 107 & 87 & 79 & 70 \\
\hline Subject 7 & $9.2(97 \%)$ & $9.0(35 \%)$ & $5.75(67 \%)$ & 133 & 100 & 87 & 83 & 74 \\
\hline
\end{tabular}

Difference in average Superior range $=25 \mathrm{deg}$.

Difference in average Inferior range between both helmets without $\mathrm{DCM}=6 \mathrm{deg}$.

Difference in average Inferior range between Shuttle with DCM and I-Suit without DCM = 15 deg 


\section{LOWER TORSO DESIGN IMPROVEMENTS}

Improved Hip/Thigh Design

The lower torso design improvements began with the conceptual design of an improved hip/thigh mobility joint system. The goal of the improved hip/thigh was to provide a more compact 2 bearing hip system while providing a more natural walking motion over the existing 2 bearing hip system.

The new 2 bearing hip system consists of a reduced angle in the hip/thigh wedge element which effectively causes the single axis mobility joint between the hip and leg bearing to be fully flexed in the neutral standing position. A new single axis convoluted joint was added below the leg bearing to delay the onset of hip bearing rotation during walking thus eliminating leg splaying and providing a more natural walking motion.

\section{Improved Boot Design}

The original I-Suit boot design consisted of a modified commercial boot mated to a single axis patterned convoluted ankle joint. The boot also included an internal inflatable foot restraint system but did not include an integral air source or pump. The use of an off the shelf commercial boot, combined with the significant axial loads to which the boot can be subjected, resulted in a large external bracket assembly which was not optimal for boot performance.

The improved boot design was a 'clean slate' look at improving I-Suit boot performance. ILC Dover teamed with a commercial boot manufacturer to provide a wellbalanced design team consisting of dedicated footwear and spacesuit designers. The team reexamined each critical boot subcomponent including outsole, midsole, insole, boot upper, heel counter, axial restraint integration, and foot indexing system.

Critical features of the outsole that were evaluated were sole material for abrasion resistance and tread design for improved traction and foot stability. The midsole features were evaluated for shock absorbance and foot stability. The upper was evaluated primarily for anthropometric fit, use of heel counter, and robust integration of load bearing axial restraint lines.

The improved boot design included development and integration of improved foot indexing concepts for testing. The two concepts that were picked for fabrication and evaluation were an improved internal inflatable system and a magnetic foot restraint.

The new boot design retained the original I-Suit ankle joint, and has shown excellent performance. Testing of the new boot and improved foot indexing system are ongoing. A picture of the new boot design is shown in Figure 5.

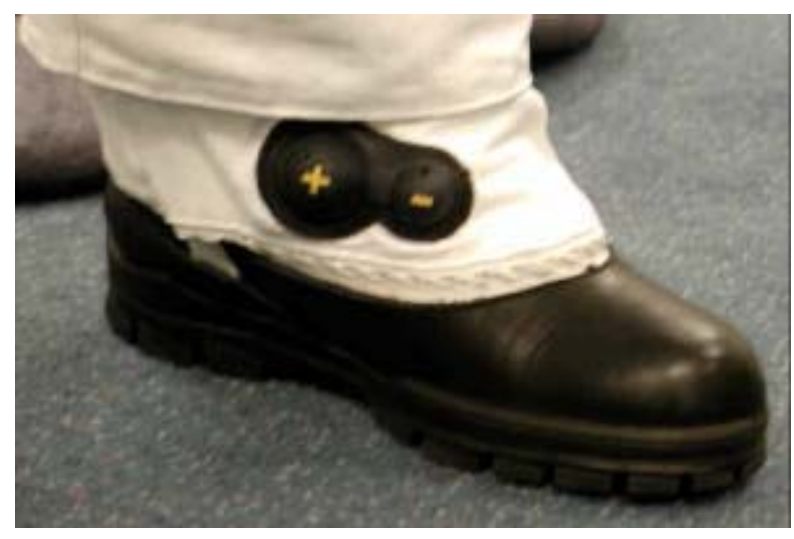

FIGURE 5

Waist Hardware

The Generation II I-Suit includes an improved latch on the waist entry body seal closure. This improved latch is a smaller, streamlined design, which incorporates a triple latching mechanism for improved safety and single motion latch engagement.

A new waist ring was also designed to allow for a modular lower torso system. Unlike the original I-Suit, the Generation II I-Suit has a waist ring, which allows the waist mobility joint to be separated from the brief. This allows different waist and brief designs to be easily integrated into the I-Suit for test and evaluation. The new waist ring also provides hard point attachment for future EVA spacesuit hardware such as safety tethers and tools.

\section{SMALL I-SUIT}

The Small I-Suit was developed to evaluate the flexibility of the I-Suit basic architecture. The goal of the Small ISuit was to evaluate the performance of small subjects utilizing the same size hardware as large subjects. The success of this performance evaluation would provide rational for one common set of suit hardware. This includes helmet, bearings, body seal closure, and waist ring.

The suit components developed for the Small I-Suit are a small size Soft Upper Torso (SUT), smaller wedge convolute shoulder mobility joint, shorter lower arms, and small size boots. The SUT bearing breadth was reduced and the distance from the helmet to the BSC was reduced. The small SUT utilized the large scye bearings, large BSC, and existing advanced I-Suit helmet. The wedge convolute shoulder mobility joint is a new design which incorporates a static wedge element at the scye bearing and a single axis balanced convoluted joint at the arm bearing. The wedge convolute shoulder joint utilized the existing large scye and arm bearings. The small lower arm was shorter in length and smaller in diameter than the existing large lower arm. The small lower arm interfaces with the large arm bearing and standard suit side wrist disconnect. Small size boots were fabricated based on the latest improved I-Suit boot design. 
Testing was performed with a small female astronaut to compare the performance of the small I-Suit to the performance of the large Shuttle Extravehicular Mobility Unit (EMU). These tests included comparative range of motion within the critical reach envelope around the suits. This testing showed the I-Suit to be equal to or better than the EMU in overall reach. See Figures 6,7 ,and 8 .

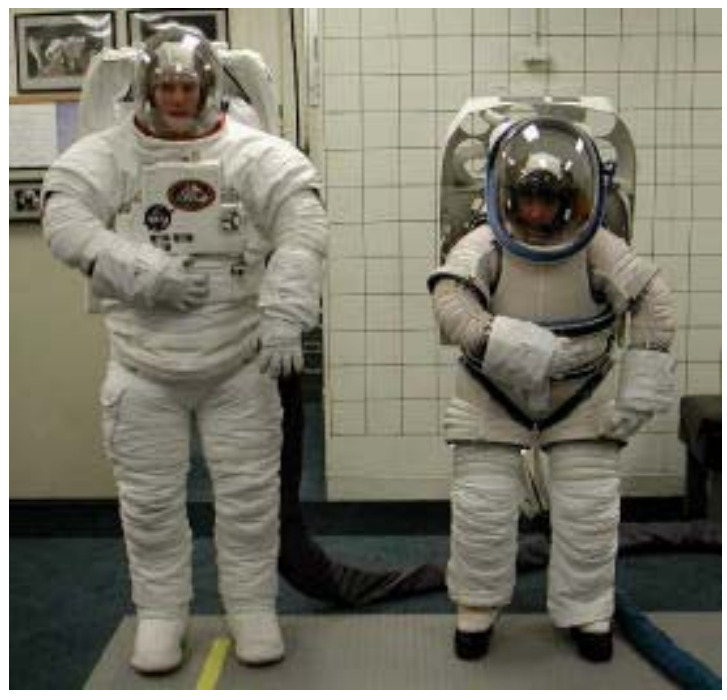

FIGURE 6

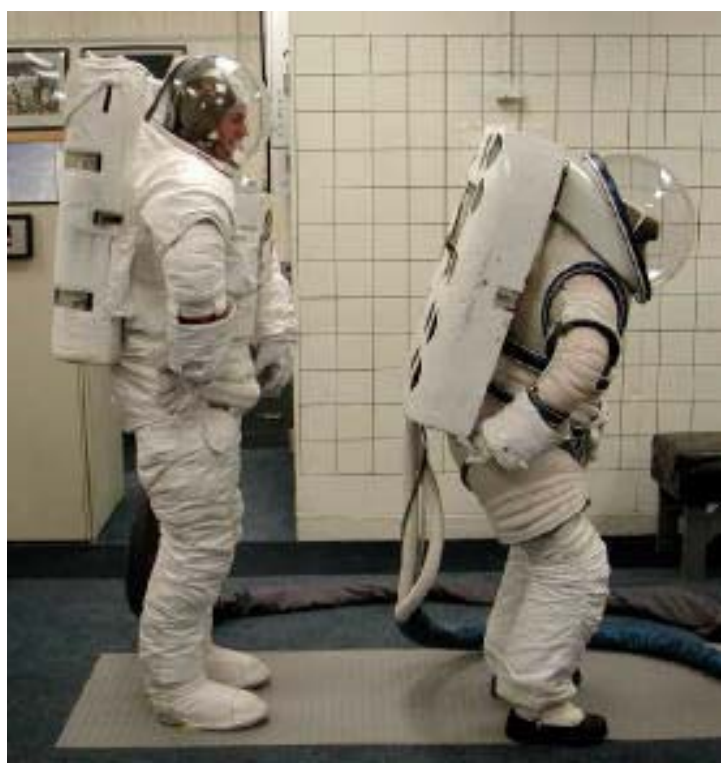

FIGURE 7

The small I-Suit and large EMU were also compared for horizontal and vertical reach. This test was performed with a large diameter circular section of space station handrail. The subjects were tasked with rotating the circular handrail section in a horizontal and vertical plane to compare reach. This test simulates handrail translation that is performed regularly during EVA activities. A picture of the handrail reach test is shown in Figure 9.

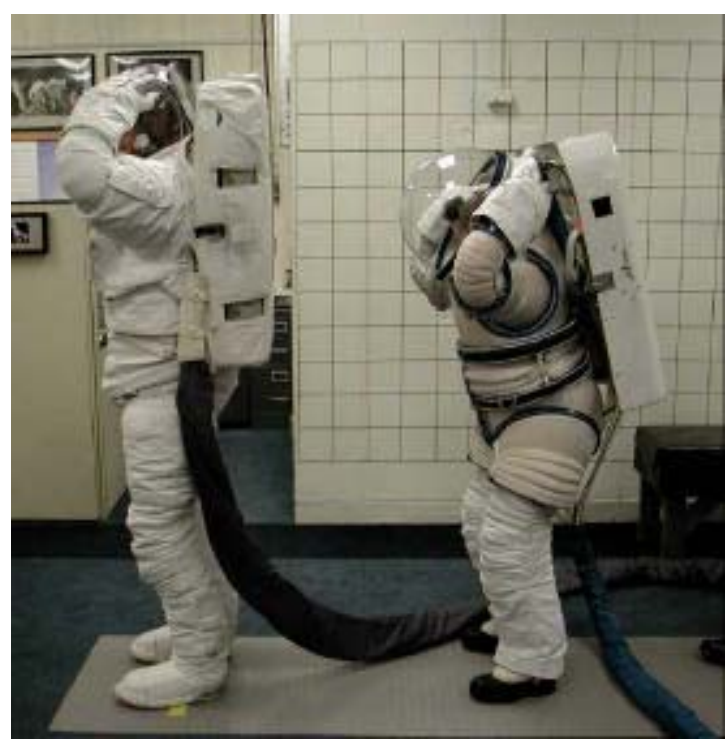

FIGURE 8

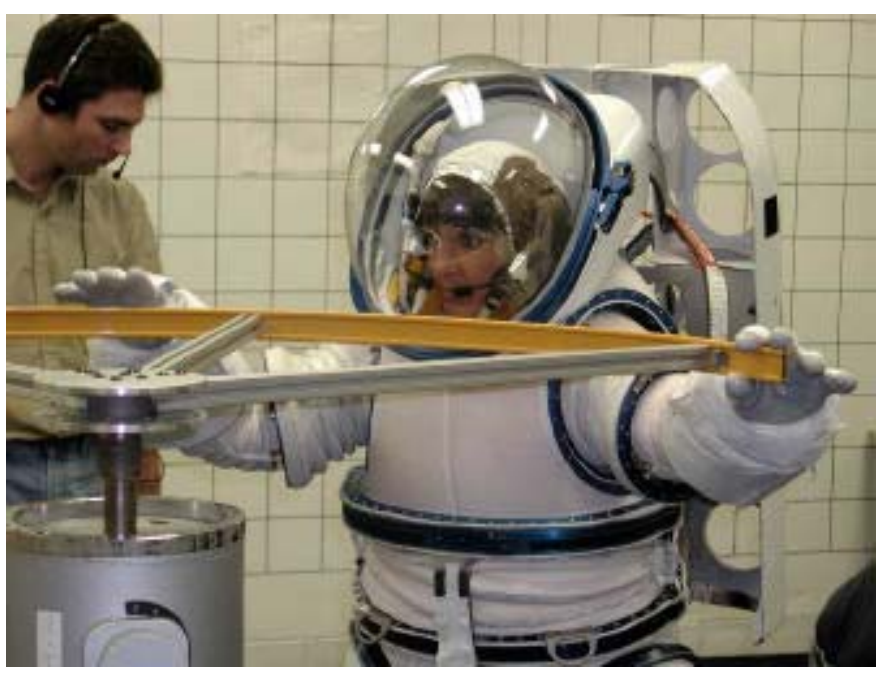

FIGURE 9

A ratcheting activity was performed to compare system level mobility and reach. Both the large EMU and small I-Suit subjects were locked in a portable foot restraint at the same distance from the workstation. The subjects then used an EVA ratchet tool to install and remove bolts. Even though the large EMU subject had 12 inches of height and 8 inches of arm reach advantage, the small I-Suit subject was able to perform the ratcheting task with equal or greater ease. When ratcheting to the side of the suit, the small I-Suit provided reduced effort and fatigue in comparison to the large EMU primarily due to the wedge convolute shoulder and hip bearings. The large EMU utilizes a single axis balanced gored shoulder joint and waist bearing.

The final test was a helmet visibility test. This test consisted of standing upright in the suits, looking up and looking down, and attempting to read numbers from scales positioned above and below the subjects. The numbers on the lower scale indicated the distance from the space suit boots the subject could see. The numbers on the upper scale indicated the distance 
behind the helmet the subject could see. The new I-Suit helmet design provided significant improvements in superior and inferior visibility over the Shuttle EMU (See Figure 10).

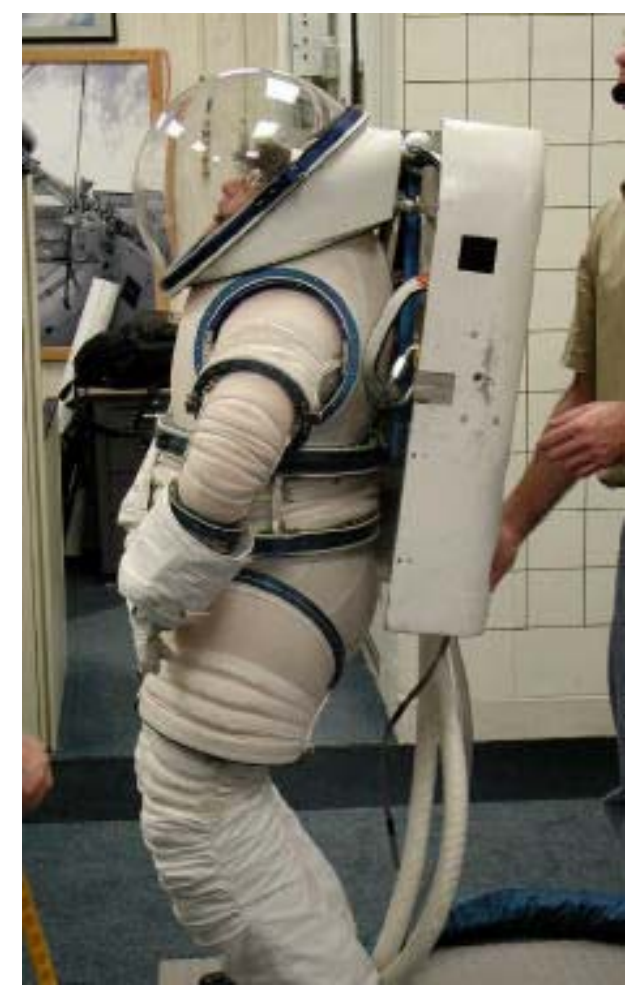

FIGURE 10

\section{ADVANCED INFORMATION TECHNOLOGIES}

Electronic Textiles were evaluated for application to EVA spacesuits. E-Textiles are new technologies that include textile fibers that are electrically conductive and pressure sensitive. Potential applications of E-Textiles include control of PLSS functions, helmet lights, helmet camera, as well as many other applications such as heater and environmental sensor control.

A pressure sensitive E-textile technology was specifically evaluated for use in the Generation II I-Suit. This technology is primarily used as a textile switch. The textile switch is equivalent to the thickness of common embroidery and is flexible, robust, and can be laundered. Two specific applications were evaluated on the Generation II I-Suit. These applications were control of helmet-mounted lights and control of a robotic rover. To evaluate information display and manipulation, the ETextile technology was coupled with the integration of a Micro-Optical head mounted display and an onboard computer, which supported a Windows operating environment.

An experiment was developed to incorporate all the elements of advanced information display and manipulation. A remote controlled rover was outfitted with an onboard video camera (See Figure 11). The rover radio controlled transmitter was integrated into the I-Suit EVA glove utilizing E-Textile switches for on/off, forward, reverse, and turning functions. The I-Suit was outfitted with a suit digital video camera, a compact computer mounted in the suit backpack, and a trackball computer-pointing device. A Micro-Optical brand headmounted optical display was mounted inside the advanced I-Suit helmet. LED helmet lights were also installed, with control being provided by the same ETextile switch technology used for the rover transmitter as seen in Figure 12.

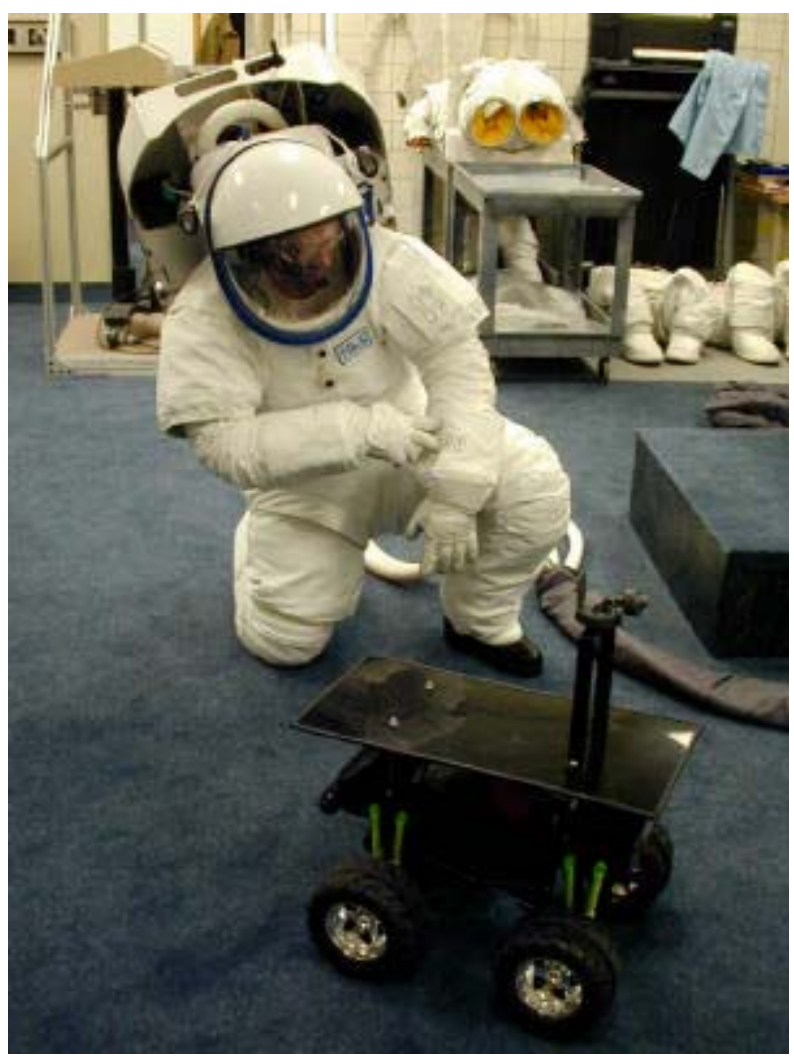

FIGURE 11

Pressurized manned testing was performed in a lab environment to evaluate the new technologies. Rover control was demonstrated using the E-Textile transmitter switches. While driving the rover, the rover camera video was transmitted wirelessly to the Micro-Optical helmet display so that the I-Suit test subject could control

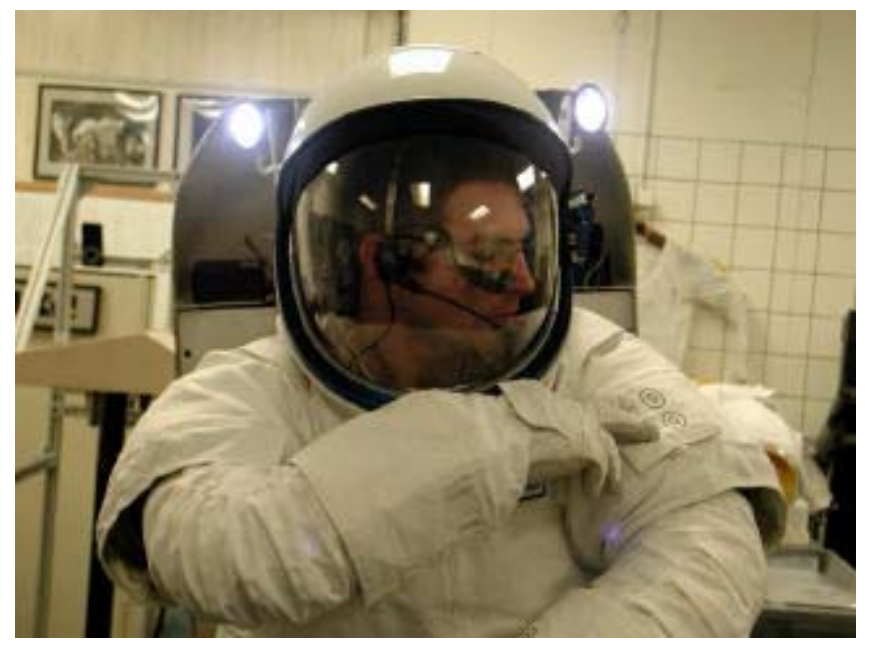

FIGURE 12 
the rover while out of direct sight. The ease of use of the optical display was also evaluated by using the pointing device to manipulate menus within a Windows operating environment to change from the rover video camera to the I-Suit video camera.

Evaluations were also performed in manipulating helmet light functions with an E-Textile switch pad. The switch pad provides the control necessary to manipulate the helmet light modes and functions.

\section{CONCLUSION}

The I-Suit development began as an EVA suit mobility demonstrator. With the completion of the Generation II ISuit, the system has evolved into a highly mobile soft EVA spacesuit integrated with technologies which allow for human robotic interaction and information acquisition. The Generation II I-Suit has also demonstrated the flexibility in sizing the I-Suit architecture. Manned pressurized testing has proven the retention of all the ISuit's mobility features while accommodating subjects from $5^{\text {th }}$ percentile female to $95^{\text {th }}$ percentile male with minimal softgoods changes. Having a highly adaptive architecture allows a common design to be enabled for a suit capable of launch and entry, zero gravity EVA, and partial gravity EVA. This type of highly mobile and flexible architecture will be a critical key to allowing crewmembers to work more effectively on increasingly more complex missions.

\section{REFERENCES}

TBD 